\title{
NONLINEAR ANALYSIS OF COMMERCIAL AIRCRAFT IMPACT ON A REACTOR BUILDING - COMPARISON BETWEEN INTEGRAL AND DECOUPLED CRASH SIMULATION
}

\author{
A.Siefert ${ }^{1}$, F.-O.Henkel ${ }^{1}$ \\ ${ }^{1}$ Woelfel Beratende Ingenieure GmbH + Co. KG, 97204 Hoechberg, Germany \\ E-mail of corresponding author: siefert@woelfel.de
}

\begin{abstract}
Since $9 / 11$ the crash of a commercial airplane on the reactor building of a nuclear power plant is a realistic design scenario. Before that the structural behaviour under a crash of a military plane was investigated by a procedure using load-time functions [1]. Thereby the computation of the load-time-function was based on a conceptional model considering the main stiffness parts and masses by discrete elements. With respect to the homogeneous structural setup of a military plane the application of this model and the derived load-time-function applied as lumped load case seems very feasible. Contrary thereto the structural setup of a commercial airplane, with e.g. the high mass concentration of the turbine or the high stiffness of the wing box compared to other parts, is different. This can be counteracted by using a more detailed finite element (FE) model for the computation of the load-time-function and by dividing the load case for the reactor building in different main load zones.

Although this represents a more detailed investigation, the procedure of using a load-time-function still has the disadvantage to separate the real scenario into two steps. Thereby the direct interaction between the structure and the airplane including all softening effects due to material respectively structural compliances is neglected. This leads to the general conclusion that by applying load-time-functions the results are conservative compared to the real behaviour.

Due to the increased capabilities of numerical software solutions it is also possible nowadays to carry out integral crash simulations, combining all effects within one simulation. Compared to the procedure of using loadtime-functions the numerical complexity and therefore the amount of work for this integral method is increased.

Within this paper both procedures (load-time function by detailed FE-model and the integral method) are exemplarily compared to each other by a crash analysis of an Airbus type A320 with different velocities (80, 120 and $160 \mathrm{~m} / \mathrm{s}$ ) on simplified reactor building. The final evaluation is carried out by the maximum displacement of the reinforced concrete reactor building wall being a criterion for the integrity of the building. With respect to the results it could be shown, that the procedure using load-time-functions has limitations for the investigation of some scenarios. This could finally lead to the decision that for the design process of new nuclear power plants the application of the integral method comprehends advantages that justify the additional efforts.
\end{abstract}

\section{INTRODUCTION}

Since the mid of the 1970ies the impact of an airplane has been a standard load case for the design of nuclear power plant buildings in Germany [4]. Thereby, due to probabilistic reasons the focus was on the crash of a military plane. This in combination with the limited capabilities of numerical simulation at that time led to an analysis procedure consisting of the following two steps:

1. Definition of the load-time-function of a military plane, following the method of Riera [1]

2. Simulation of loading the structure with the load-time-function lumped over the impact area

Hence due to this separation of load determination and its application, this represents a decoupled investigation of the real scenario. Following this procedure the direct interaction between structure and airplane is neglected. In reality, especially the compliance of the structure would cause a softening of the impact leading to lower values of the peak load. Accordingly a general conclusion is that the results are conservative compared to the real crash behaviour. 
Since the incident of 9/11 the crash of a commercial aircraft on structure also plays [5] an important role in the design of new nuclear power plants. Due to the fact that the construction design of a commercial airplane is different from that of a military plane, the analysis procedure applied so far is reviewed by the work presented here. Thereby the main focus is on the computation and application of the load-time-function as load case. Accordingly, avoiding any limitations due to the aircraft model, the load-time-function is computed by the same FE-model which is used for the integral crash simulation. Consequently all investigations are carried out with the explicit solver of the finite element program ABAQUS [2].

The review presented here is divided into two steps. After the setup of the A320 FE-model the load-timefunction is computed by the crash of the complete model on a rigid wall with 80,120 and $160 \mathrm{~m} / \mathrm{s}$. As an alternative, all main parts, i.e. the turbines, the wings and the fuselage, are crashed separately to consider the structural differences of the commercial aircraft. Afterwards the partial load time functions are summed up to an integral value, whereby the different distance to the wall is considered by a time offset. Finally, both methods of computing the load-time-function are compared to each other demonstrating the problems of computing an adequate load-timefunction for a commercial airplane structure.

In the second part of the paper a crash on a simplified reactor building model of a nuclear power plant is computed by using the load-time-function, derived by the separated approach, and the integral procedure. The evaluation of both simulations is carried out by the maximum displacement as a criterion for the integrity of the reactor building. Finally, the results are compared to each other and advantages respectively disadvantages are presented.

\section{MODEL SETUP OF COMMERCIAL AIRCRAFT A320}

The first step for the crash analysis is the detailed model setup of the commercial aircraft. During the last years models of different aircraft types have been developed by Woelfel . Starting point was the modelling of the F4 - Phantom as the simulation results could be proven by the experimental data of the Sandia test [3]. Subsequently, models of A320, B747, B767 and A380 were generated. Thereby the procedure follows the modelling of passenger cars for crash tests in the automotive sector. The main difference consists in the model precision, which is due to the aircraft size and due to the fact that the simulation aims at the evaluation of the reactor building and not of the aircraft. Consequently, only the main parts, describing the structural behaviour and representing the global mass distribution, are considered as shown in the following list:

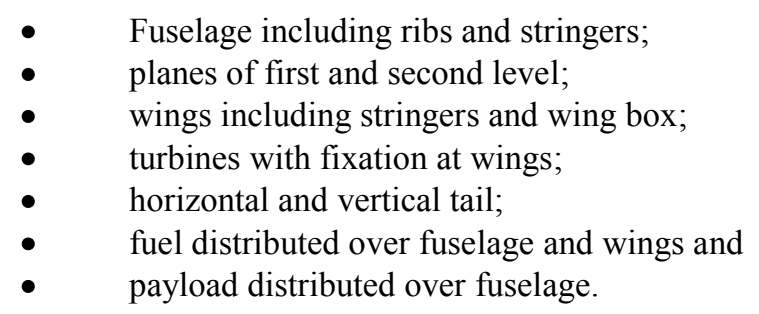

Within this study the plane type A320 is used, as it is widely spread and hence represents a standard load case for almost all nuclear power plants worldwide. The geometric information for the setup of the FE model is taken from published CAD data. The following figures show a complete view and a cross section in the symmetry plane of the model: 

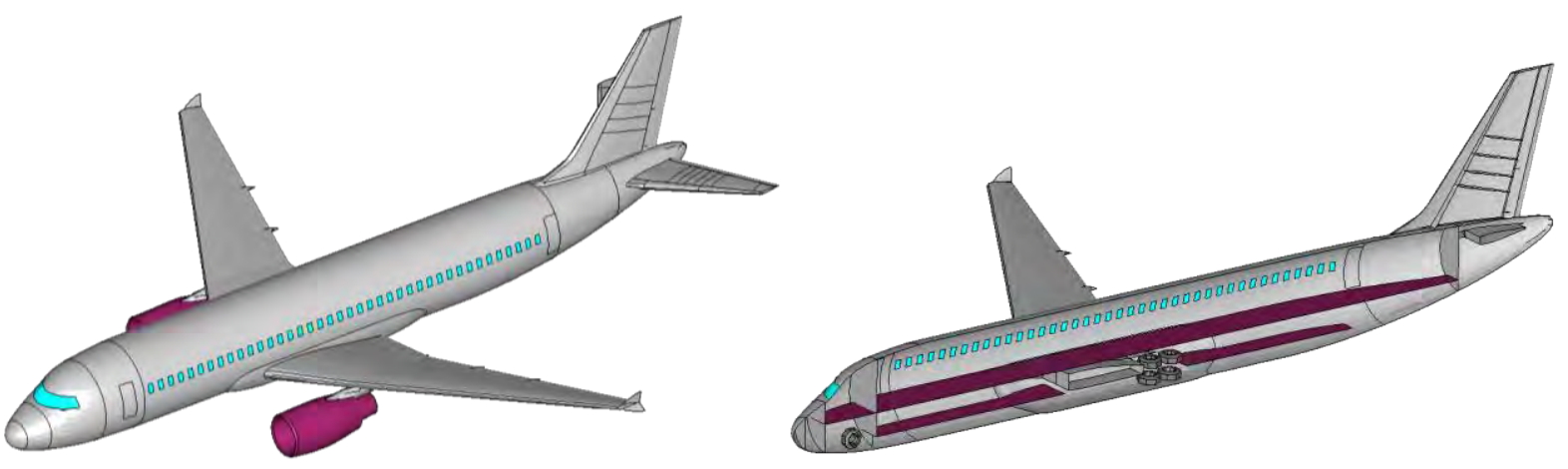

Fig. 1: Finite-Element model of Airbus type A320

The model setup consists of shell and beam elements for the structural parts and of discrete mass elements for the fuel and the payload. In summary the model includes about 45,000 elements and 170,000 degrees of freedom. The material definition reflects the nonlinear and strain dependent behaviour and failure defined by maximum strains and stresses. Although the Airbus A320 was one of the first commercial aircraft using carbon- and glass-fibre materials the main structural parts are made of high-strength aluminium. As no detailed information of the stress-strain behaviour of the materials, used for the A320, are known, standardised materials of aeronautical engineering are considered. For all materials applied the standard values of their densities are used, while the fuel mass and the payload are modelled by the discrete mass elements, positioned with respect to the real distribution. The total mass of the model is about $76.5 \mathrm{t}$ representing the maximum take-off weight.

\section{COMPUTATION OF LOAD-TIME-FUNCTION}

The computation of the load-time-function is carried out with a crash on a wall (size $10.0 \times 24.0 \mathrm{~m}$ ) defined as a rigid body. The support of the wall is fixed for all degrees of freedom at its reference point, which is also used for the evaluation of the load-time-function. The interaction between the wall and the aircraft and the aircraft to itself is defined by a general contact formulation using a penalty approach including friction. The simulation is carried out with 80,120 and $160 \mathrm{~m} / \mathrm{s}$ for the complete model and for the separated parts, i.e. the turbines, the wings and the fuselage. Based on the theory of an ideal plastic impact the results of the load-time-function finally are checked by the principle of momentum (Equation 1).

$$
d P=d m \cdot v_{0}=\sum F \cdot d t
$$

Figure 2 shows the initial and the final state of the simulation with the complete model and a velocity of $160 \mathrm{~m} / \mathrm{s}$. Although the velocity of $160 \mathrm{~m} / \mathrm{s}$ is twice high as the landing velocity, the aircraft is not completely destroyed. Accordingly it must be mentioned that due to the modelling of the fuel by discrete mass elements, an explosion is not included in the simulation. Beside that it can be observed that the turbines and the wings are completely destroyed and that they are ruptured form the fuselage.

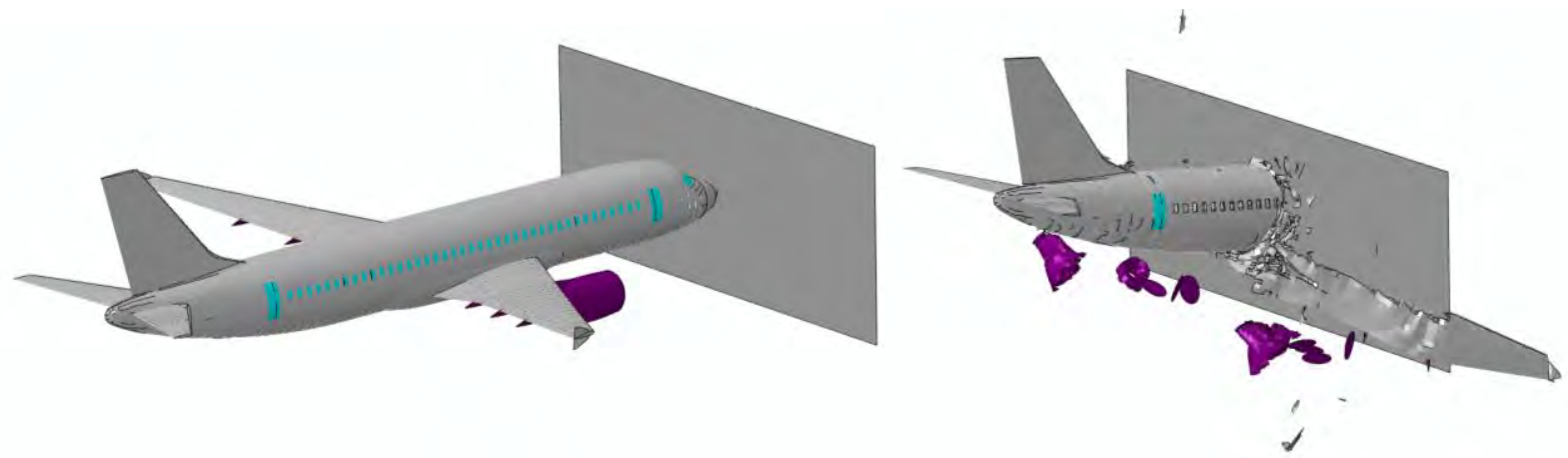

Fig. 2: Simulation of crash onto rigid wall of A320 with initial velocity of $160 \mathrm{~m} / \mathrm{s}$ 
Similar behaviour can be determined for the other two velocities, but for $80 \mathrm{~m} / \mathrm{s}$ it has to be emphasized that the wings are almost not impacting the wall and furthermore are not ruptured from the fuselage.

In figure 3 the results of the load-time-function are presented for the velocity of $80 \mathrm{~m} / \mathrm{s}$ with the complete model. Due to sensitivities with the impact on an infinite stiff body the curve progression of the initial results includes numerical artefacts. Accordingly, the results are revised with two smoothing algorithms ('Polynomial Method' and 'Straight Average Method'). While the 'Polynomial Method' is an analytical approximation by the order of 20, the 'Straight Average Method' is a numerical approximation. Thereby each curve point represents an averaged value of the defined time span $\mathrm{dt}$ before and after (here e.g. for $\mathrm{dt}=0.002,0.005$ and $0.01 \mathrm{~s}$ ).

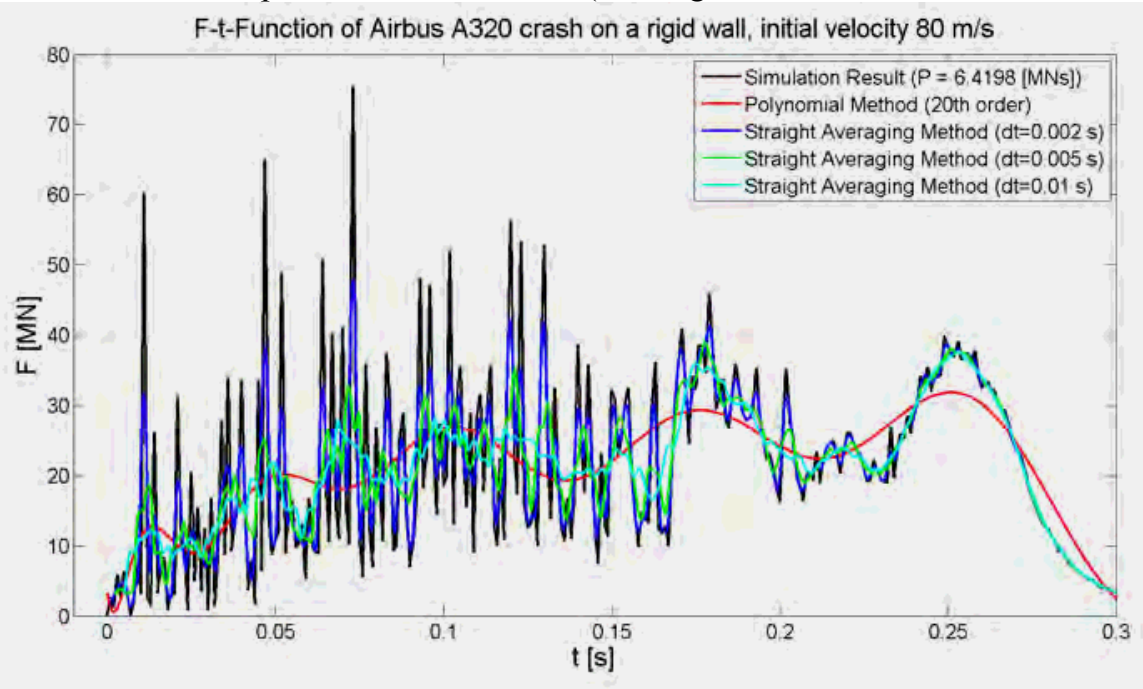

Fig. 3: Load-time-function values of A320 crash onto rigid wall with initial velocity of $80 \mathrm{~m} / \mathrm{s}$

The smoothed results ('Straight Average Method') of all velocities are presented in figure 4. Proving them by the balance of momentum, it can be determined that the difference to the analytical values is less than $10 \%$. This is theoretically based on the characteristic of the impact, which is not completely plastic. A visual confirmation therefore is the elastic rebound observed at the end of the simulation, which finally leads to an increased value of the momentum.

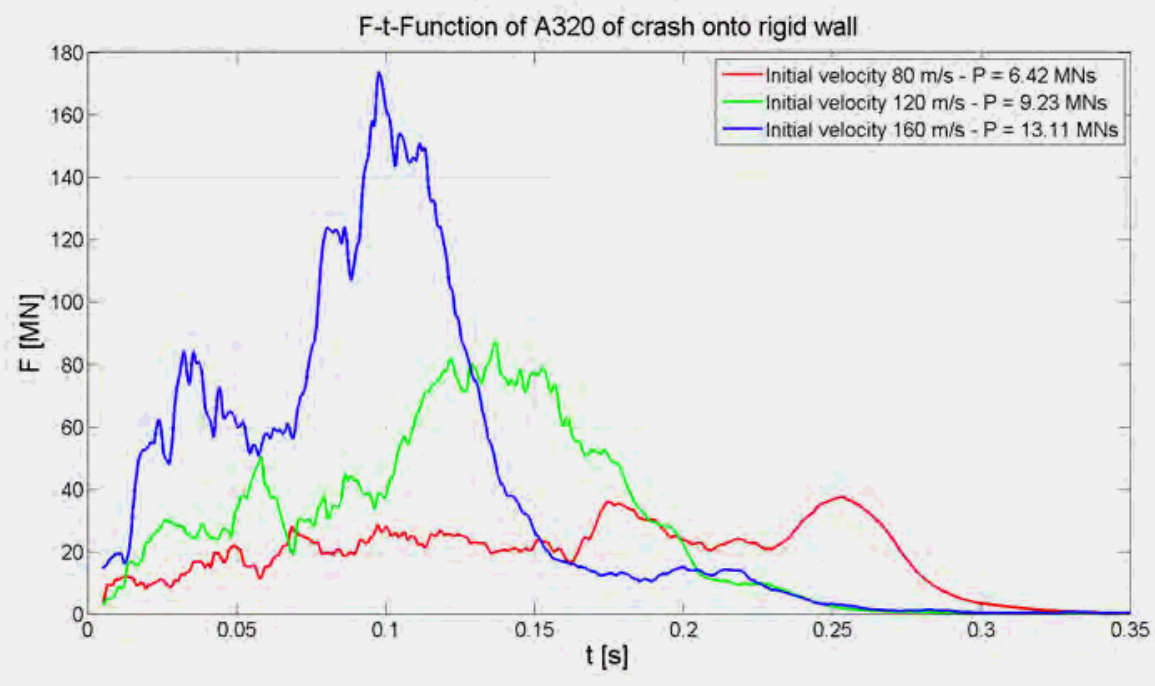

Fig. 4: Load-time-function of A320 crash onto rigid wall with different initial velocities

As shown by the plot the influence of the initial velocity on the peak force is strong, as the increase from 80 to $160 \mathrm{~m} / \mathrm{s}$ has a factor of four. Furthermore, it must be emphasized that the characteristics of the load-timefunctions are changing with respect to the velocity. 
For considering the structural difference of a commercial airplane the original procedure by using a global load-time-function can be divided. Thereby the load-time-function is computed separately for each main part (turbine, wing and fuselage) by a crash on a rigid wall, and for the simulation with the reactor building it is finally applied on the corresponding load area.

Before this final step, the crash results on the rigid wall for the global and the divided procedure are compared to each other. Accordingly the results of the divided procedure are summed up. Clarifying the procedure for computing the summed results, it has to be mentioned that for each part (turbines, wing and fuselage) the crash on the rigid wall is simulated separately. Afterwards the sub-load-time-functions are summed up considering the time span between first contact at the aircraft tip and the structural part (see figure 5).

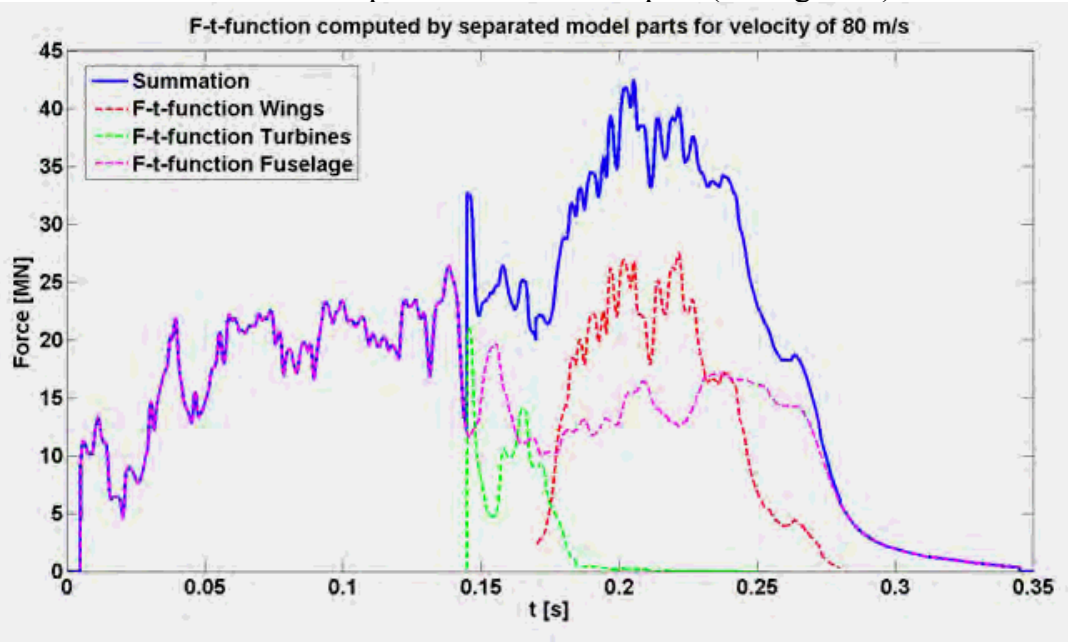

Fig. 5: Load-time-function of A320 crash as summation of separated crash analysis for $80 \mathrm{~m} / \mathrm{s}$

Comparing now the results of the complete model with those of the separated analysis (figure 6) it is primarily observed that the characteristics are very similar for both procedures, i.e. in a large part the curves are in the same range. An exception thereto are the maximum peak values for 120 and $160 \mathrm{~m} / \mathrm{s}$, which are almost $40 \%$ higher for the separated simulation than for the complete model. The reason for this is the interaction between the model parts enabling a flux of the initial kinetic energy, which does not exist in the case of separated approach.

This can easily be proven by looking at the velocity e.g. of the turbines within the simulation using the complete model. The initial value of the velocity is reduced immediately after the first impact of the fuselage, although the turbines are not in contact with the wall. Accordingly a part of the kinetic energy of the turbines is transferred to the fuselage and therefore is reducing the peak values of the turbine itself. Consequently, the approach of using separated load-time-functions for the main parts to consider the structural differences of a commercial aircraft does not represent the real behaviour correctly.

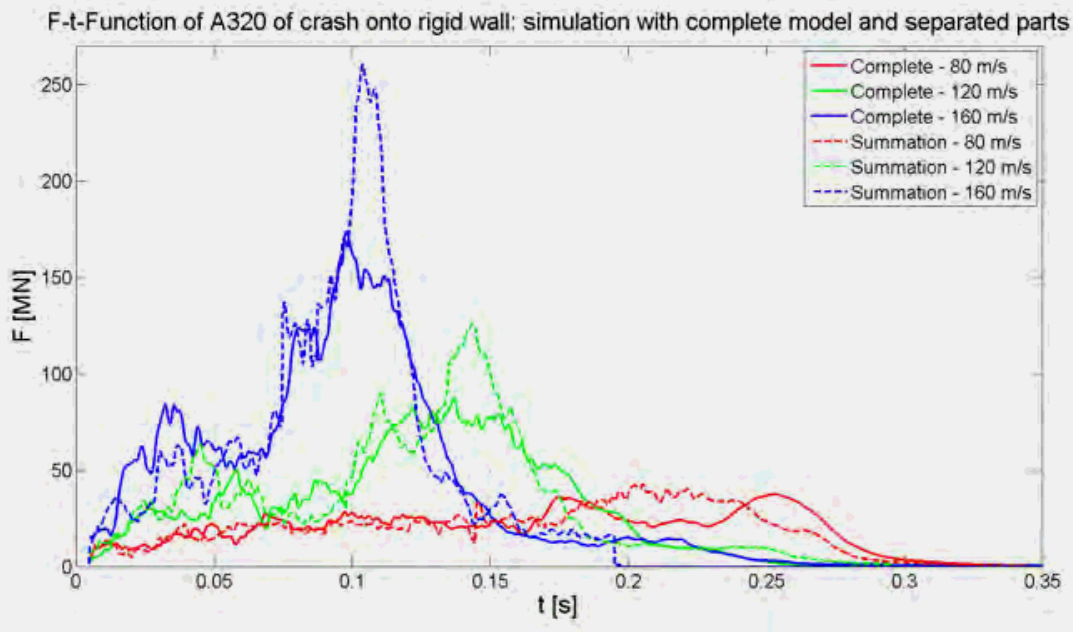

Fig. 6: Load-time-function of A320 crash as summation of separated and complete analysis 
Furthermore, it has to be mentioned that a homogenous distribution by using the load-time-function of the complete model is also different to the real crash, as local maximum loads are lumped over the whole contact area. This conclusion is based on the maximum load per area values of the different structural parts. While the maximum load with values of 20 to $25 \mathrm{MN}$ is similar for the turbines, the wings and the fuselage, the areas are pretty different, with values of $4 \mathrm{~m}^{2}$ for the turbine, $7 \mathrm{~m}^{2}$ for the wing and $13 \mathrm{~m}^{2}$ for the fuselage. This in combination with the less conservative results regarding the peak values leads to the decision to use the separated approach for the final investigation of the reactor building.

\section{MODEL SETUP OF REACTOR BUILDING}

The integral and the decoupled simulations are carried out on an exemplary reactor building, see figure 7, defined via cylindrical shape at the bottom (diameter $30.0 \mathrm{~m}$ ) and at hemisphere at the top (complete height $60.0 \mathrm{~m}$ ). The wall consists of reinforced concrete with a constant thickness of $1.8 \mathrm{~m}$. While the concrete is modelled via volume elements the reinforcement is implemented by truss elements for the shear part and by membrane elements for the bending part. The area of the reinforcement represents standard values of new nuclear power plants. Altogether the model consists of 580,000 nodes and 520,000 elements.
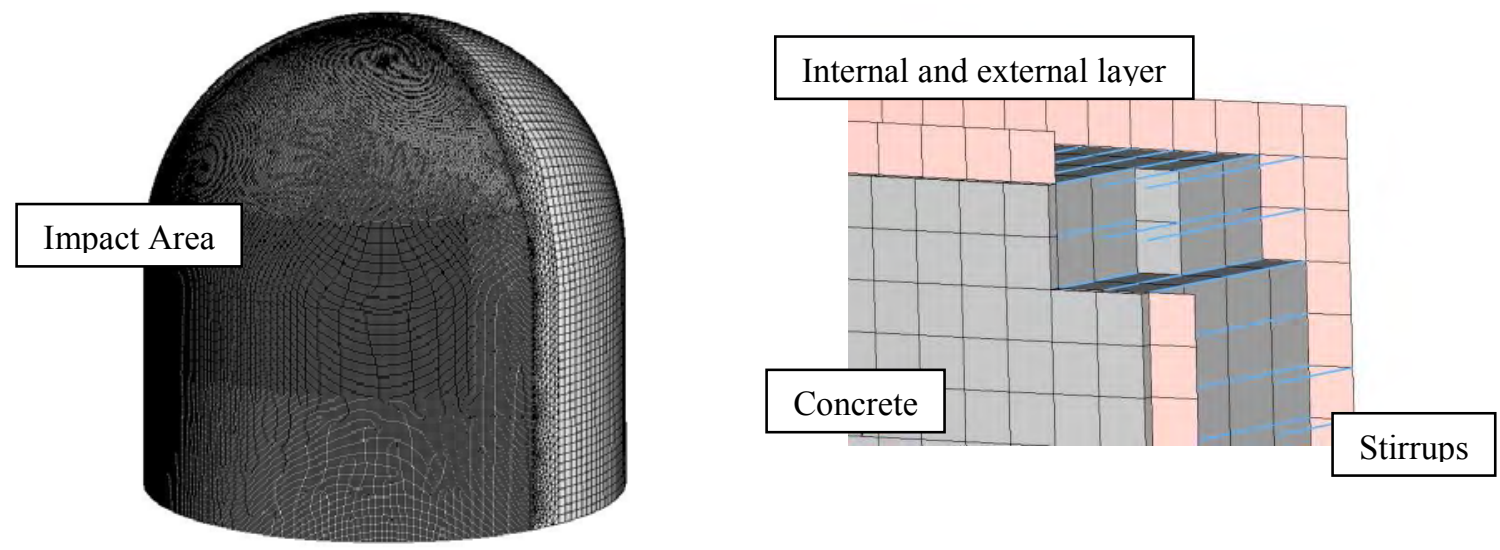

Fig. 7: Simplified reactor building and model detail for reinforcement

With respect to compute the damage of the reactor building due to the aircraft impact, the material behaviours are defined nonlinear and have a failure criteria. Furthermore, the strain rate dependence influencing the peak resistance is implemented. Representing current designs a concrete of type C45/55 and a steel of type BSt 500 are chosen.

\section{CRASH ANALYSIS OF A320 ON REACTOR BUILDING}

For the complete simulation the impact area is defined on the upper part of the cylinder. The load areas for using the results of the load-time-function are defined by a straight projection of the structural parts (turbines, wings and fuselage) on the outer surface of the reactor building (figure 8.a). The application is carried out as a pressure load normal to the surface, which is a difference to the integral simulation, where the load direction is defined by the vector of the initial velocity. This influence based on the $3 \mathrm{D}$ characteristic of building is amplified for real scenarios by the slope angle of the flight path. The time of both simulations is specified with $0.3 \mathrm{~s}$ for the initial velocity of $80 \mathrm{~m} / \mathrm{s}$ and with 0.25 for 120 and $160 \mathrm{~m} / \mathrm{s}$. In figure 8 the final state is shown for integral simulation with $160 \mathrm{~m} / \mathrm{s}$. The destruction of the airplane is similar to the simulation for the crash on the rigid wall, i.e. that for $160 \mathrm{~m} / \mathrm{s}$ with the complete airplane being destroyed, except the back part of the fuselage. For 80 and $120 \mathrm{~m} / \mathrm{s}$ only the frontal part of the fuselage is destroyed. Furthermore, small damage can be observed for the wings and the turbines. In both cases the turbines are ruptured from the wings, but the wings are still connected to the fuselage. 

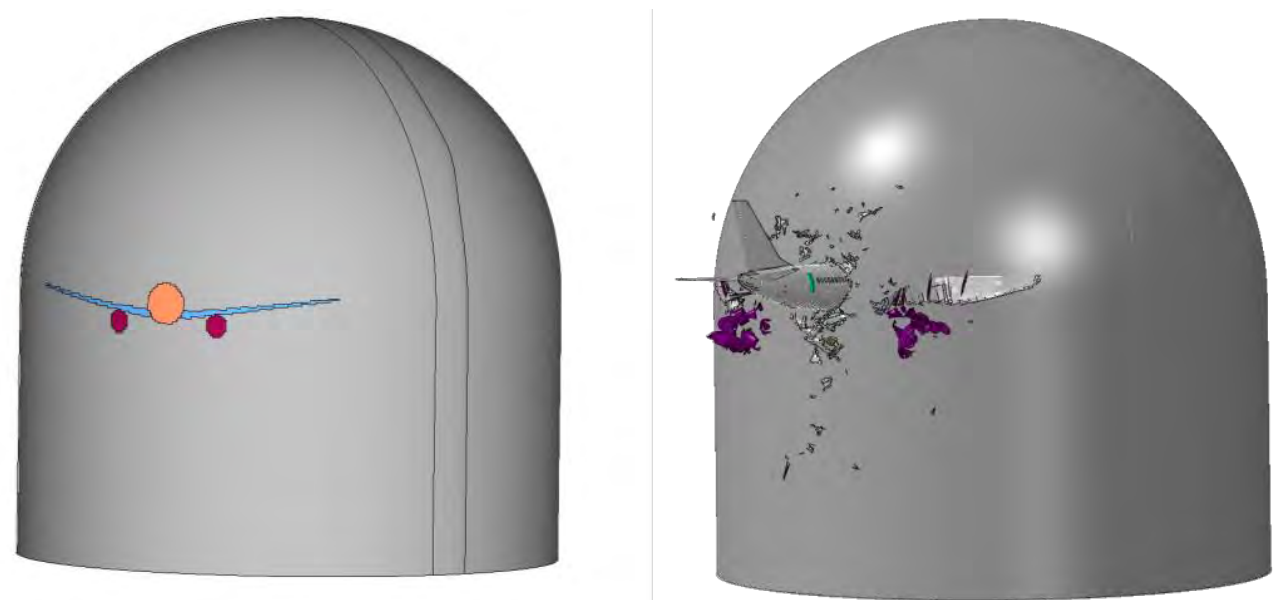

Fig. 8.a: Load area distribution for using separated load-time-functions (left)

Fig. 8.b: Final state of integral simulation with initial velocity of $160 \mathrm{~m} / \mathrm{s}$ (right)

The final evaluation of the simulation is carried out by the maximum displacement in the area of the initial contact. As already mentioned the simulation includes elastic effects, accordingly the maximum displacement occurring right after the maximum load is finally decreased. Therefore, the following table 1 - beside the maximum value - also shows the result at the end of the simulation. The values of the integral simulation are higher for all simulations, which is especially apparent in the case of $160 \mathrm{~m} / \mathrm{s}$. The reason for this difference can be separated into two effects.

\begin{tabular}{|c|c|c|c|c|}
\hline \multirow{2}{*}{ Initial Velocity $[\mathrm{m} / \mathrm{s}]$} & \multicolumn{2}{|c|}{ Load-Time-Function Approach } & \multicolumn{2}{c|}{ Integral Approach } \\
\cline { 2 - 5 } & Maximum [cm] & Final [cm] & Maximum [cm] & Final [cm] \\
\hline 80 & 0.4 & 0.4 & 0.9 & 0 \\
\hline 120 & 1.4 & 0.5 & 5.0 & 3.4 \\
\hline 160 & 3.0 & 0.5 & 17.1 & 13.1 \\
\hline
\end{tabular}

Tab. 1: Maximum and final value of displacement in the area of the fuselage impact

On the hand the above-mentioned interaction within the model enables for the integral approach the flux of kinetic energy from the wings and the turbines to the fuselage. This increases the load of this area, finally leading to higher displacements of the reactor building or higher destruction respectively. This is demonstrated in the following figure by showing the velocity change of one node at the turbines for all velocities. As can be seen, the value of the initial velocities right after starting the simulation is decreased, although the turbine itself is not in contact with the building. This effect is physically plausible as obviously all structural parts are affected if one part is decelerated. One important fact thereby is, that depending on the initial velocity and the structural design this effect is different.

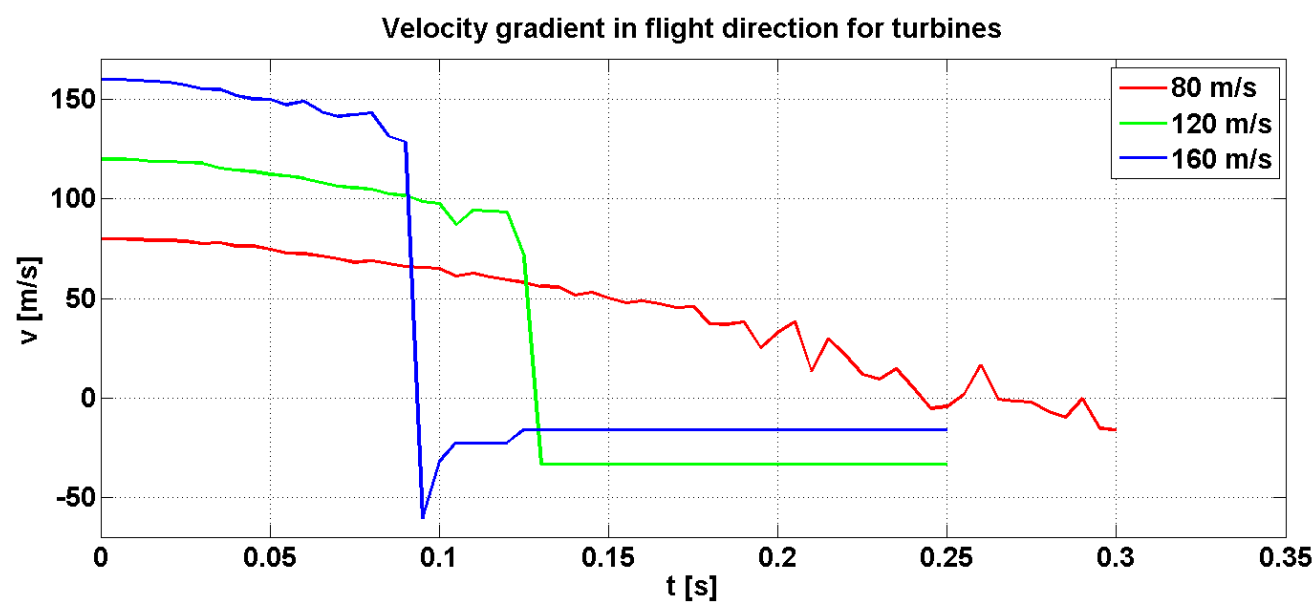

Fig. 9: Progression of velocity at turbines over simulation time for all initial velocities 
On the other hand the local load distribution has a big influence on the final results. Although in the simulation using load-time-function a differentiation is made for the structural main parts, as the turbines, wings and the fuselage, the load is lumped within these areas. The following figure 10 shows the distribution for compressive stresses in the flight direction for an initial velocity of $160 \mathrm{~m} / \mathrm{s}$ at $\mathrm{t}=0.025$ and $\mathrm{t}=0.08 \mathrm{~s}$.
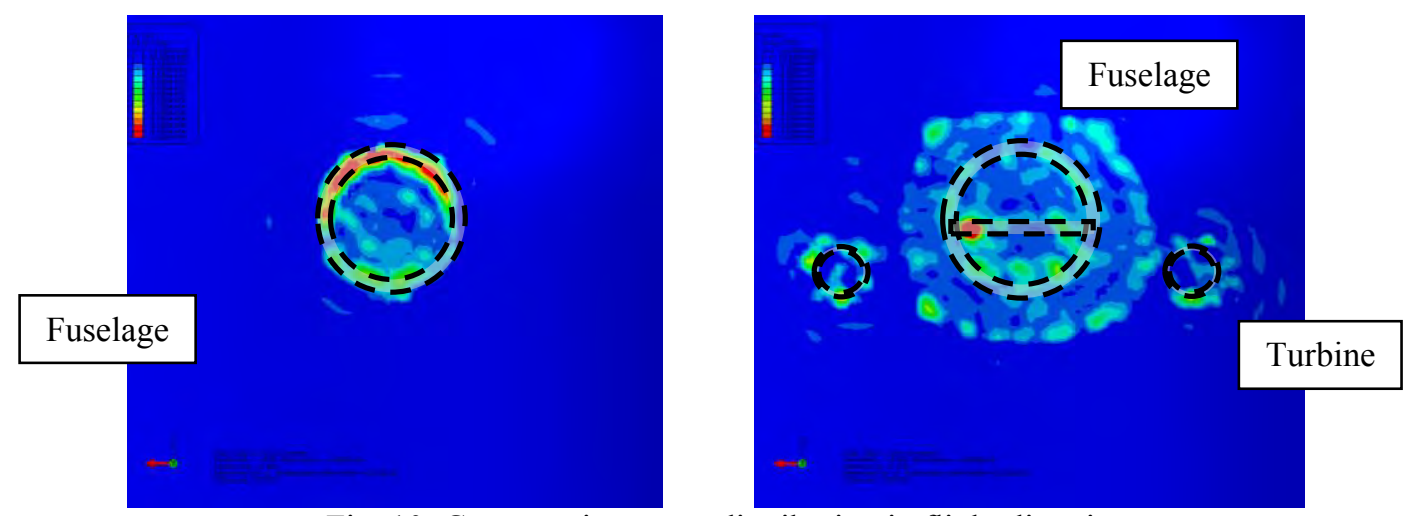

Fig. 10: Compressive stress distribution in flight direction

As shown by the figure, the stress distribution is not homogenous. It represents rather the structural setup, i.e. the maximum values are in the area of structural parts with high stiffness as the intermediate floor and the envelope of the fuselage or the case of the turbines. Compared to the load-time-function approach with a lumped stress distribution the values are strongly increased, leading to greater local destruction and respectively higher displacement values.

\section{CONCLUSION}

The work here presented showed a comparison between using load-time-functions or an integral simulation approach for the crash analysis of a commercial aircraft on a reactor building. Avoiding any differences due to the modelling, both simulations used a detailed FE-model of the airplane type Airbus A320. The analysis is carried out for three initial velocities, namely 80,120 and $160 \mathrm{~m} / \mathrm{s}$.

Based on the results of the maximum displacement it could be observed that for the simulation of a commercial airplane using the load-time-function shows lower values than for the integral approach. This counteracts the general assumption that results by using the load-time-function are in general conservative because softening effects are neglected. The differences of using the load-time-function are caused by two effects.

The first one is the decoupled procedure, whereby energetic fluxes within the airplane are not considered in the final crash simulation. The second one is the fact, that the load-time-function is always lumped over the complete contact area. Although structural differences are considered by using the divided load-time-function procedure, the final load is applied on the complete area of this structural part. This could not reflect local stress concentrations in the area of the stiffer parts as e.g. the envelope of the fuselage. Accordingly, the load values and following the destruction of the reactor building are lower.

\section{REFERENCES}

[1] RIERA, J.D.: "On the Stress Analysis of Structures Subjected to Aircraft Impact Forces", Nuclear Engineering and Design, Vol. 8, pp 415-426, 1968.

[2] ABAQUS (Software), Version 6.11-1, ABAQUS Inc., Providence, USA, www.abaqus.com

[3] VON RIESEMANN W.A. et al.: "Full-Scale Aircraft Impact Test for Evaluation of Impact Forces, Part1: Test Plan, Test Method and Test Results", Proceedings of the $10^{\text {th }}$ international conference on SMiRT, Los Angeles, USA, 1989

[4] HENKEL, F.-O., WÖLFEL, H.-P.: "Building Concepts against Airplane Crash", Journal of Nuclear Engineering and Design, Volume 79, pp. 397-409, 1984

[5] HENKEL, F.-O., KLEIN, D.: "Variants of Analysis of the Load Case Airplane Crash", Proceedings of the $19^{\text {th }}$ international conference on SMiRT, Toronto, Canada, August 2007 